\title{
Autosomal recessive mendelian susceptibility to mycobacterial diseases due to a partial deficiency
}

INSERM

\section{Source}

INSERM. (1999). Orphanet: an online rare disease and orphan drug data base. Autosomal recessive mendelian susceptibility to mycobacterial diseases due to a partial deficiency. ORPHA:319539

Autosomal recessive mendelian susceptibility to mycobacterial disease (MSMD) due to a partial deficiency describes a group of genetic variants of MSMD (see this term) due to autosomal recessive mutations in the IFNGR1 and IFNGR2 genes which lead to a residual response of IFN-gamma. 